\title{
Mapping chemical bonding of reaction intermediates with femtosecond X-ray laser spectroscopy
}

\author{
Ph. Wernet ${ }^{1}$, M. Beye ${ }^{1}$, F. de Groot ${ }^{2}$, S. Düsterer ${ }^{3}$, K. Gaffney $^{4}$, S. Grübel ${ }^{5}$, R. Hartsock ${ }^{4}$, F.
} Hennies $^{6}$, I. Josefsson ${ }^{7}$, B. Kennedy ${ }^{6}$, K. Kunnus ${ }^{1}$, T. Leitner ${ }^{1}$, T. Mazza ${ }^{8}$, M. Meyer ${ }^{8}$, D. Nordlund ${ }^{9}$, M. Odelius ${ }^{7}$, W. Quevedo ${ }^{5}$, P. Radcliffe ${ }^{8}$, I. Rajkovic ${ }^{5}$, B. Schlotter ${ }^{10}$, M. Scholz ${ }^{5}$, S. Schreck ${ }^{1}$, E. Suljoti $^{1}$, S. Techert ${ }^{5}$, J. Turner ${ }^{10}$, C. Weniger ${ }^{1}$, W. Zhang ${ }^{4}$, A. Föhlisch ${ }^{1}$

\footnotetext{
${ }^{1}$ Helmholtz-Zentrum Berlin GmbH, Albert-Einstein-Str. 15, 12489 Berlin, Germany

${ }^{2}$ Department of Inorganic Chemistry and Catalysis, Utrecht University, Utrecht, Netherlands

${ }^{3}$ DESY, Notkestr. 85, 22670 Hamburg, Germany

${ }^{4}$ PULSE Institute, SLAC National Accelerator Laboratory, Menlo Park, CA 94025 USA

${ }^{5}$ Max Planck Institute for Biophysical Chemistry, Am Faßberg 11, 37077 Göttingen, Germany

${ }^{6}$ MAX IV Laboratory, Lund, Sweden

${ }^{7}$ Department of Physics, Stockholm University, AlbaNova University Center, Stockholm, Sweden

${ }^{8}$ European XFEL GmbH, Albert-Einstein-Ring 19, 22761 Hamburg, Germany

${ }^{9}$ SSRL, SLAC National Accelerator Laboratory, Menlo Park, CA 94025 USA

${ }^{10}$ LCLS, SLAC National Accelerator Laboratory, Menlo Park, CA 94025 USA
}

\begin{abstract}
We determine the pathways in the photo-dissociation reactions of $\mathrm{Fe}(\mathrm{CO})_{5}$ both in the gas phase and in solution by mapping the valence electronic structure of the reaction intermediates with femtosecond X-ray laser spectroscopy.
\end{abstract}

\section{Introduction}

Molecular structure and bonding determine the dynamic pathways of molecules in their multidimensional landscapes and thus define the outcome of chemical reactions. Characterizing chemical bonding in short-lived reaction intermediates is hence the key to understanding chemical selectivity.

Here we present our time-resolved X-ray view of a molecular dissociation reaction. We address photo-dissociation of the prototypical transition-metal carbonyl molecule $\mathrm{Fe}(\mathrm{CO}) 5$ both in the gas phase and in solution (Figure 1) [1]. How chemical bonding evolves during these reactions is largely unknown and the reaction pathway is debated in solution [2-4]. We use X-ray laser spectroscopy to map the electronic structure during dissociation with femtosecond time resolution [5]. This gives direct access to chemical bonding, spin and oxidation state of the metal center in the reaction intermediates and complements structural probes [6]. As we probe the same observables in gas phase and in solution our data enable a critical assessment of the role of the solvent in $\mathrm{Fe}(\mathrm{CO})_{5}$ photodissociation. 


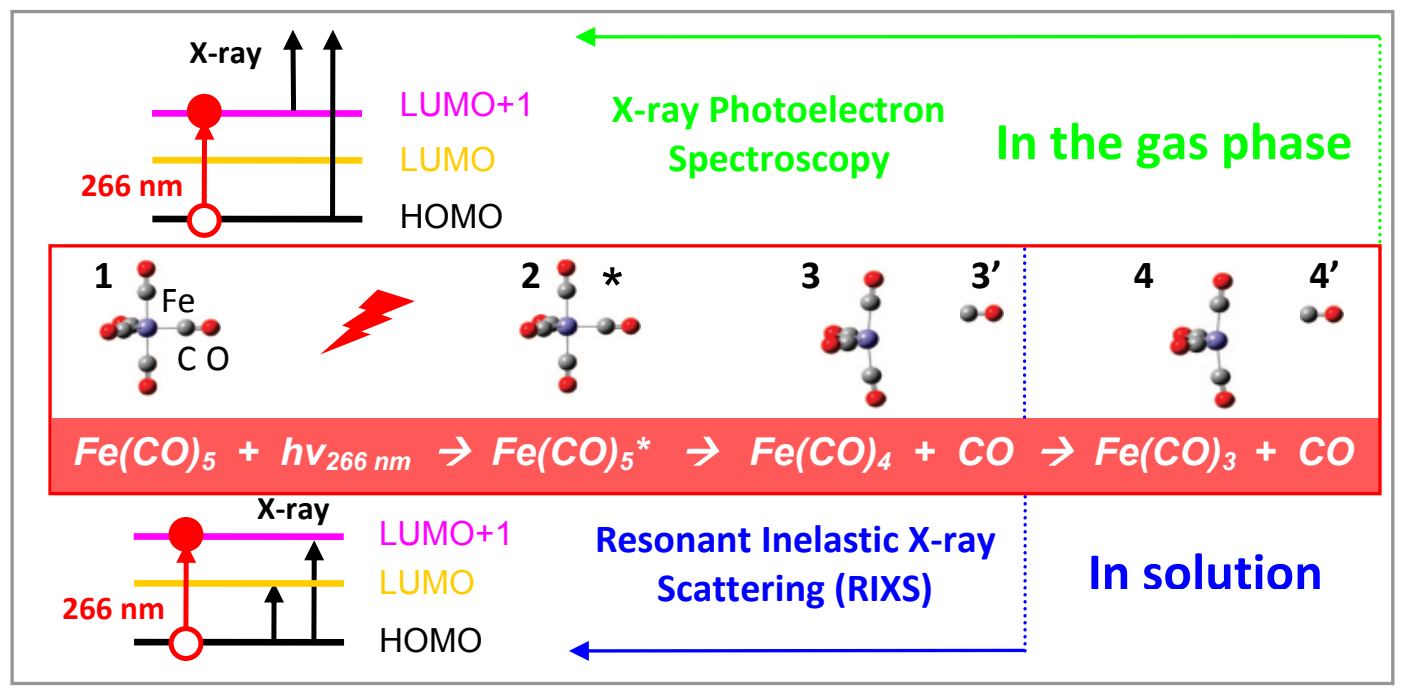

Fig. 1. Simplified scheme for the photo-dissociation reaction of $\mathrm{Fe}(\mathrm{CO})_{5}$ in the gas phase and in solution upon pumping at $266 \mathrm{~nm}$ (middle) [1] with labels 1-4': 1 ground state $\mathrm{Fe}(\mathrm{CO})_{5}, 2$ photoexcited $\mathrm{Fe}(\mathrm{CO})_{5}, \mathbf{3} \mathrm{Fe}(\mathrm{CO})_{4}, \mathbf{3}$ ' photo-dissociated $\mathrm{CO}, \mathbf{4} \mathrm{Fe}(\mathrm{CO})_{3}$ and $\mathbf{4}$ ' thermally ejected $\mathrm{CO}$. In solution, the reaction stops at $\mathbf{3} / \mathbf{3}^{\prime}$. We probe the evolution of occupied levels with time-resolved Xray photoelectron spectroscopy in the gas-phase (top) and of valence excitations by measuring photon energy losses with time-resolved inelastic X-ray scattering (RIXS) in solution (bottom).

\section{Experimental}

$\mathrm{Fe}(\mathrm{CO})_{5}$ molecules were pumped at $266 \mathrm{~nm}$ and probed at defined time delays with X-ray pulses from the X-ray free electron laser sources FLASH in Hamburg, Germany, for the gas-phase reaction and from the Linac Coherent Light Source (LCLS) in Stanford, USA, for the solution reaction (concentration $1 \mathrm{~mol} / 1$ in ethanol). In the gas phase, valence band (Figure 2, left), core $3 \mathrm{p}$ and resonant core-to-valence $3 p \rightarrow 3 d$ photoelectron spectra (not shown) were measured. In solution, RIXS at the Fe L-edge was used as a probe of the valence electronic structure (Figure 2, right). The temporal resolution in both experiments amounted to $300 \mathrm{fs}$ given by the jitter between optical and $\mathrm{X}$-ray lasers. Multi-configurational ab initio calculations of the X-ray spectra were performed to identify and characterize in detail the reaction intermediates.

\section{Results}

A selection of our femtosecond time-resolved X-ray laser spectroscopy results is shown in Figure 2. The differential gas-phase valence-band photoelectron spectra (Figure 2A) display positive intensities arising from the appearances of $\mathrm{CO}$ and photoproducts and negative intensities due to the depletion of $\mathrm{Fe}(\mathrm{CO})_{5}$. By accounting for $\mathrm{Fe}(\mathrm{CO})_{5}$ depletion we deduce the valence-band photoelectron spectrum of $\mathrm{Fe}(\mathrm{CO})_{4}$ at a delay of $1 \mathrm{ps}$. This shows how, compared to $\mathrm{Fe}(\mathrm{CO})_{5}$, the $\mathrm{Fe}$ $3 \mathrm{~d}$ levels are shifted and the shape of the $\mathrm{CO}$ molecular bands are modified by the loss of one $\mathrm{CO}$ ligand. This information on chemical bonding complements structural information from electron diffraction [6]. The $\mathrm{CO}$ peak (Figure 2B) at short delays rises in a step-like fashion due to $\mathrm{CO}$ dissociation from $\mathrm{Fe}(\mathrm{CO})_{5}\left(3^{\prime}\right.$ ' in Figure $\left.2 \mathrm{~B}\right)$ and it increases exponentially for later delays (4' in Figure $2 \mathrm{~B}$ ) due to thermal $\mathrm{CO}$ desorption from vibrationally hot $\mathrm{Fe}(\mathrm{CO})_{4}$. This is direct evidence for the sequential CO loss postulated in [2]. With our RIXS approach we probe for the first time a chemical reaction in solution with femtosecond RIXS. The data shown in Figure $2 \mathrm{C}$ display the valence excitations probed locally and in an element- and site-selective way at the Fe center of 
$\mathrm{Fe}(\mathrm{CO})_{4}$. Comparison to theory (not shown) shows that $\mathrm{Fe}(\mathrm{CO})_{4}$ is in a singlet state in contrast to [3] and in agreement with [4]. According to our data (Figure 2D and calculations) $\mathrm{Fe}(\mathrm{CO})_{5}$ is excited to a 1 MLCT state $(1 \rightarrow 2)$ and relaxes within our time resolution to a $1 \mathrm{LF}$ state $(2 \rightarrow 3)$ with subsequent dissociation to singlet $\mathrm{Fe}(\mathrm{CO})_{4}$. In contrast to the gas phase the excess energy in $\mathrm{Fe}(\mathrm{CO})_{4}$ is dissipated to the solvent and no further $\mathrm{CO}$ is lost. Details on chemical bonding, spin and oxidation state as inferred from a comparison to our calculations will be presented in addition.
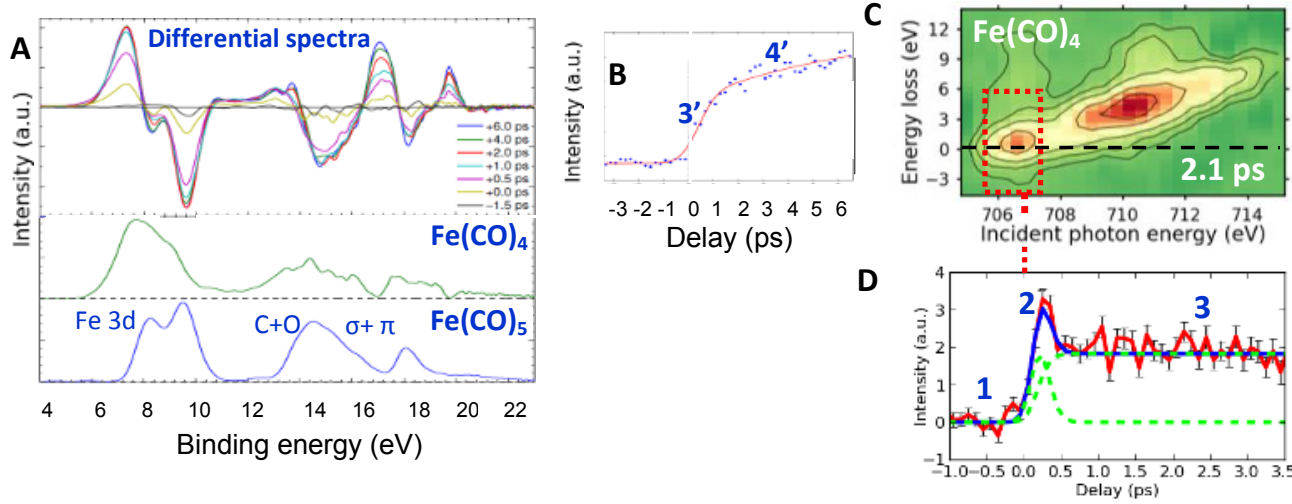

Fig. 2. Femtosecond time-resolved $\mathrm{X}$-ray laser spectra mapping $\mathrm{Fe}(\mathrm{CO})_{5}$ dissociation in the gasphase (A, B) and in solution (C-D). A: Gas-phase valence-band photoelectron spectra of, from top to bottom, $\mathrm{CO}, \mathrm{Fe}(\mathrm{CO})_{5}$ for various pump-probe delays (differential spectra), $\mathrm{Fe}(\mathrm{CO})_{4}$ (calculated from the differential spectrum at $1 \mathrm{ps}), \mathrm{Fe}(\mathrm{CO})_{5}$ with the $\mathrm{Fe} 3 \mathrm{~d}$ and the $\mathrm{C}$ and $\mathrm{O} \sigma$ and $\pi$ molecular orbitals. B: Intensity of the CO peaks vs. delay (circles) with a fit curve (line, step function plus exponential increase). C: RIXS spectra (energy loss vs. incident photon energy tuned to the Fe Labsorption edge) of solvated $\mathrm{Fe}(\mathrm{CO})_{5}$ and $\mathrm{Fe}(\mathrm{CO})_{4}$ (taken at $2.1 \mathrm{ps}$ ), respectively. D: Intensity of the marked region vs. delay (red) and a fit curve (blue, Gaussian plus step function).

\section{Acknowledgements}

Portions of this research were carried out on the SXR Instrument at the Linac Coherent Light Source (LCLS), a division of SLAC National Accelerator Laboratory and an Office of Science user facility operated by Stanford University for the U.S. Department of Energy. The SXR Instrument is funded by a consortium whose membership includes the LCLS, Stanford University through the Stanford Institute for Materials Energy Sciences (SIMES), Lawrence Berkeley National Laboratory (LBNL), University of Hamburg through the BMBF priority program FSP 301, and the Center for Free Electron Laser Science (CFEL).

\section{References}

1. M. Poliakoff and J. J. Turner, Angew. Chem. Int. Ed. 40, 2809 (2001).

2. S. A. Trushin, W. Fuss, K. L. Kompa and W. E. Schmid, J. Phys. Chem. A 104, 1997 (2000).

3. P. T. Snee, Ch. K. Payne, S. D. Mebane, K. T. Kotz and C. B. Harris, J. Am. Chem. Soc. 123, 6909 (2001).

4. B. Ahr, M. Chollet, B. Adams, E. M. Lunny, Ch. M. Laperle and Ch. Rose-Petruck, Phys. Chem. Chem. Phys. 13, 5590 (2011).

5. Ph. Wernet, Phys. Chem. Chem. Phys. 13, 16941 (2011).

6. H. Ihee, J. Cao, A. H. Zewail, Angew. Chem. Int. Ed. 40, 1532 (2001). 